\title{
Nano-textured polymers for future architectural needs
}

\author{
Cees W.M. Bastiaansen, Albert Schenning, Michael Debije and Dirk J. Broer* \\ Department of Functional Organic Materials and Devices, Eindhoven University of Technology, \\ Eindhoven, The Netherlands
}

Received: 6 June 2013

Accepted: 7 November 2013

\begin{abstract}
The rapid developments in molecular sciences like nanotechnology and self-organizing molecular systems generate a wealth of new materials and functions. In comparison to electronics the application in architecture remains somewhat underexposed. New functionalities in optics, responsive mechanics, sensing and adjustable permeation for gases and water might add to new opportunities in providing for personal comfort and energy management in houses and professional buildings. With a number of examples we demonstrate how complex but well-controlled molecular architectures provide functionalities worthwhile of being integrated in architectural designs. Optical coatings are capable of switching colors or reflectivity, creating possibilities for design but also for the control of thermal transmission through windows. They respond to temperature, light intensity, or both. Selectively-reflective thin polymer layers or paint pigments can be designed to switch between infrared and visible regions of the solar spectrum. Coatings can be designed to change their topology and thereby their appearance, of interest for in-house light management, or just for aesthetic appeal. Plastic materials can be imbued with the property of autonomous sun tracking and provided morphing behavior upon contact with moisture or exposure to light. Many of these materials need further developments to meet the requirements for building integration with respect to robustness, lifetime, and the like, which will only be accomplished after demonstration of interest from the architectural world.
\end{abstract}

Keywords: Functional polymers, responsive coatings, smart materials, liquid crystal networks, architectural coatings

\section{Introduction}

Nanotechnology based on organic materials started a revolution with respect to new functionalities for applications in electronics, communication and medical technology. Only recently have new developments in the field of organic nanotechnology found their way to the world of civil engineering and architecture. In this respect one can imagine new solutions for the creation of green, energyneutral buildings, of in-house climate control and solutions to enhance personal comfort in houses and professional buildings like offices and hospitals. Table 1 shows a wish list that was generated from discussion sessions between architects from the Delft University of Technology and polymer engineers from the Eindhoven University of Technology (Klein, 2011).

It is the objective of this chapter to provide solutions for the desired features presented in Table 1 by means of well-constructed organic or polymer materials with organizational control down to the molecular level. By utilizing a combination of top-down and bottom-up structuring technologies, it is

\footnotetext{
${ }^{*}$ Corresponding author: Prof. Dr. Dirk J. Broer, Professor at Eindhoven University of Technology, Chemical Engineering \& Chemistry, Department of Functional Organic Materials \& Devices (SFD), Helix building STO 0.34, Den Dolech 2, 5612 AZ Eindhoven, The Netherlands. Tel.: +31 40247 5875, Mob: +31 6 51662354; E-mail: d.broer@tue.nl.
}

ISSN 2213-302X/13/\$27.50 (C) 2013 - IOS Press and the authors. All rights reserved

This article is published online with Open Access and distributed under the terms of the Creative Commons Attribution Non-Commercial License. 
Table 1

List of features fitting in the concept of the future envelope for buildings

\begin{tabular}{|c|c|}
\hline Feature & Effect \\
\hline Dynamic color & $\begin{array}{l}\text { Surfaces changing color in order to maximize or minimize energy gain; switch between heat } \\
\text { absorption and heat rejection }\end{array}$ \\
\hline Oxygen control & Sensing and regulation of $\mathrm{O}_{2}$ versus $\mathrm{CO}_{2}$ concentrations; surfaces that actively turn $\mathrm{CO}_{2}$ into $\mathrm{O}_{2}$ \\
\hline Electricity & $\begin{array}{l}\text { Surface uses sun and air currents to produce electricity; eventually to be used for autonomous } \\
\text { operating building elements }\end{array}$ \\
\hline Clean/protected & $\begin{array}{l}\text { Surface that always stays clean and is protected from pollution; surface that can be cleaned } \\
\text { remotely }\end{array}$ \\
\hline Sun/view & $\begin{array}{l}\text { Window optimizing direct, diffuse or no light transmission, while maintaining unobstructed views; } \\
\text { switchable windows }\end{array}$ \\
\hline Adaptive daylight & $\begin{array}{l}\text { Roof designed to meet lighting requirements by responding to exterior conditions, e.g. by } \\
\text { morphogenesis }\end{array}$ \\
\hline Air/Sound filter & $\begin{array}{l}\text { Air and sound should be selectively filtered through the surface. Unwanted smells and sounds are } \\
\text { kept out }\end{array}$ \\
\hline Self-healing & Surfaces and constructs are self-healing after damage or aging \\
\hline Structural integrity & $\begin{array}{l}\text { Surfaces able to allocate different degrees of stiffness locally; soft and pleasing if desired, strong } \\
\text { and tough when needed }\end{array}$ \\
\hline
\end{tabular}

possible to create complex molecular architectures that provide functions corresponding to many of the concepts that are developed for future building projects. With top-down structuring technologies we refer to the formation of (often) periodic structures down to the micrometer level by means of photolithographic processes, (inkjet) printing, photo-replication or embossing, to name some techniques. For bottom-up structuring, we are utilizing the ability of particular molecules to self-organize into complex, three-dimensional structures which can be permanently fixed by a polymerization into a densely crosslinked polymer network. An overview of various aspects of liquid crystal networks can be found in (Broer, Crawford, \& Zumer, 2011). For this purpose we often use liquid crystal monomers (an example is shown in Fig. 1) which may be aligned into complex morphologies using surface interactions, external electric or magnetic fields, or molecular properties including hydrogen-bridge formation or chirality. Based on their molecular structure, we are able to make the materials response to light in both their color and shape (Yamada, 2008), (van Oosten, 2008, 2009) to create adjustable reflection with respect to wavelength and light polarization (Broer, 1995), and to form nanoporous membranes with well controlled, adjustable pore dimensions (Luengo Gonzalez, 2008).

\section{Liquid crystals and liquid crystal networks: Molecular architectures in polymer systems}

We all know liquid crystals are used as the light switching medium in flat panel television and mobile phone displays. The principle of light switching is based on changing the state of polarization of transmitted light. This technique can also be successfully utilized in building components such as switchable windows and switchable door panels. For instance, glass panels with these properties have been brought to the market by a number of suppliers under names Privacy Window, Privacy Glass, E-Glass, and Smart Glass, among others, and are in most cases based on an electro-optical switching between a transparent and a scattering state of a liquid crystal polymer composite. What 


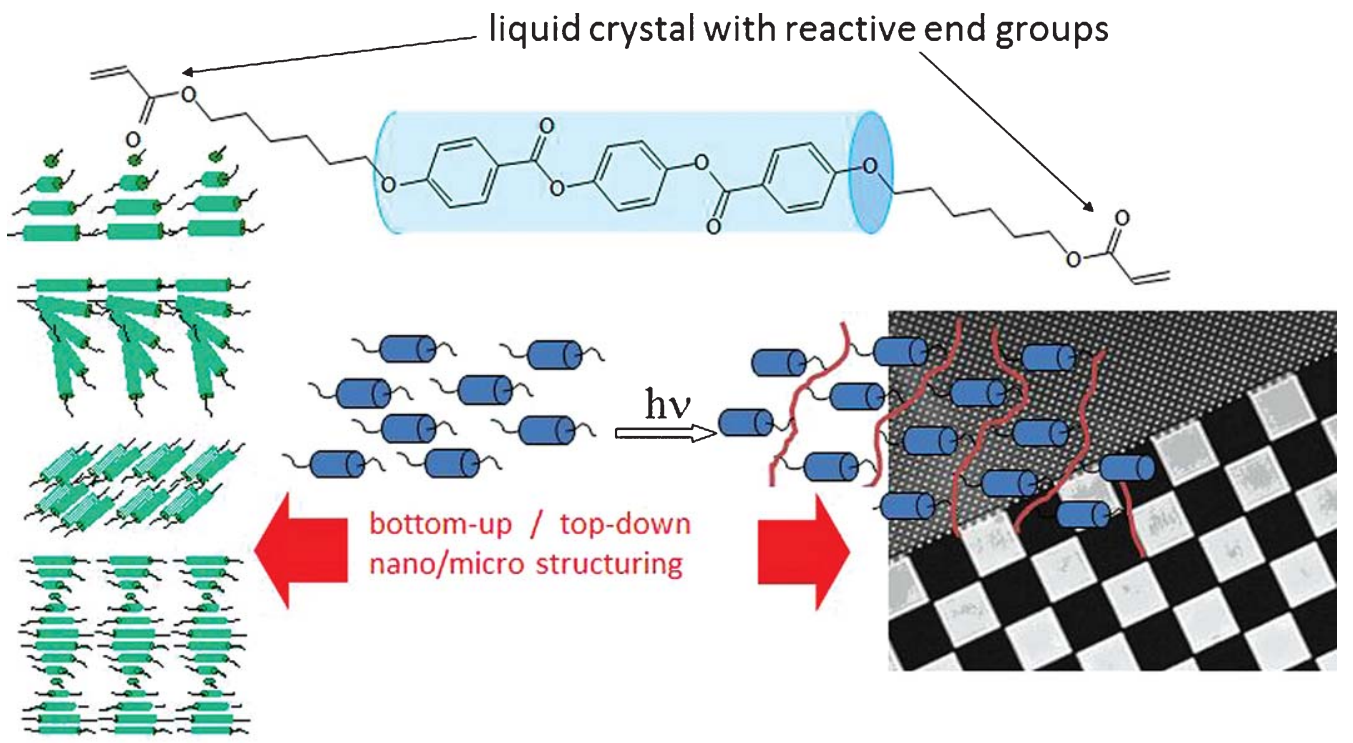

Fig. 1. Principle of photopolymerization of reactive liquid crystal monomers. The monomers can be controlled to align uniaxially, twisted, splayed, tilted or in a molecular helix (bottom-up) and can be applied locally by lithography or printing (top-down) to form a solid plastic film with control over the molecular positioning and orientation.

is less well-known is that liquid crystals can be polymerized into plastic networks that preserved the molecular order of the liquid crystals in a solid matrix. These so-called 'liquid crystal networks' exhibit extraordinary properties. For instance, as low molecular weight components in displays they are able to control the state of polarization of light, and thus the transmission of light. When the orientation of the molecules in the network is brought into a helix with a pitch of the order of the wavelength of light, they start to reflect parts of the light spectrum. This feature is illustrated in Fig. 2. By controlling the pitch of the helix, the reflection band can range from ultra violet through the visible region to the infrared region of the light spectrum. This property can be used to protect against harmful light, change the appearance of an object, or control heat uptake or heat rejection when applied at the exterior of a building or a window. It is also used to create efficient light concentrators for solar energy panels with freedom in both shape and color. In addition they are used to provide sensing functions by using changes in color or reflection band upon exposure to, among other gases, an excess of $\mathrm{CO}_{2}$ (Han, 2010). The pitch can be controlled by the presence of chiral molecules, and adjusted accurately by the concentration of chiral units. External triggers change the 'helical twisting power' of these chiral additives in a reversible way, enabling switching between reflection wavelengths. When the reflection wavelength is in the (near) infrared part of the spectrum, the material is transparent and colorless but performs its function by reflecting climate-affecting radiation.

As shown in Fig. 2, the helicoidally ordered networks can be processed as continuous films but can also be ground into smaller particles which can be added to paint as a pigment. In general, the formation of helicoidal or even more complex structures in these liquid crystal polymers can be controlled down to the molecular level. One can roughly say that by applying structure through techniques like molding, printing and lithography, the production time and production costs scale inversely with the dimension size of the structure. This makes production of larger surfaces using these 

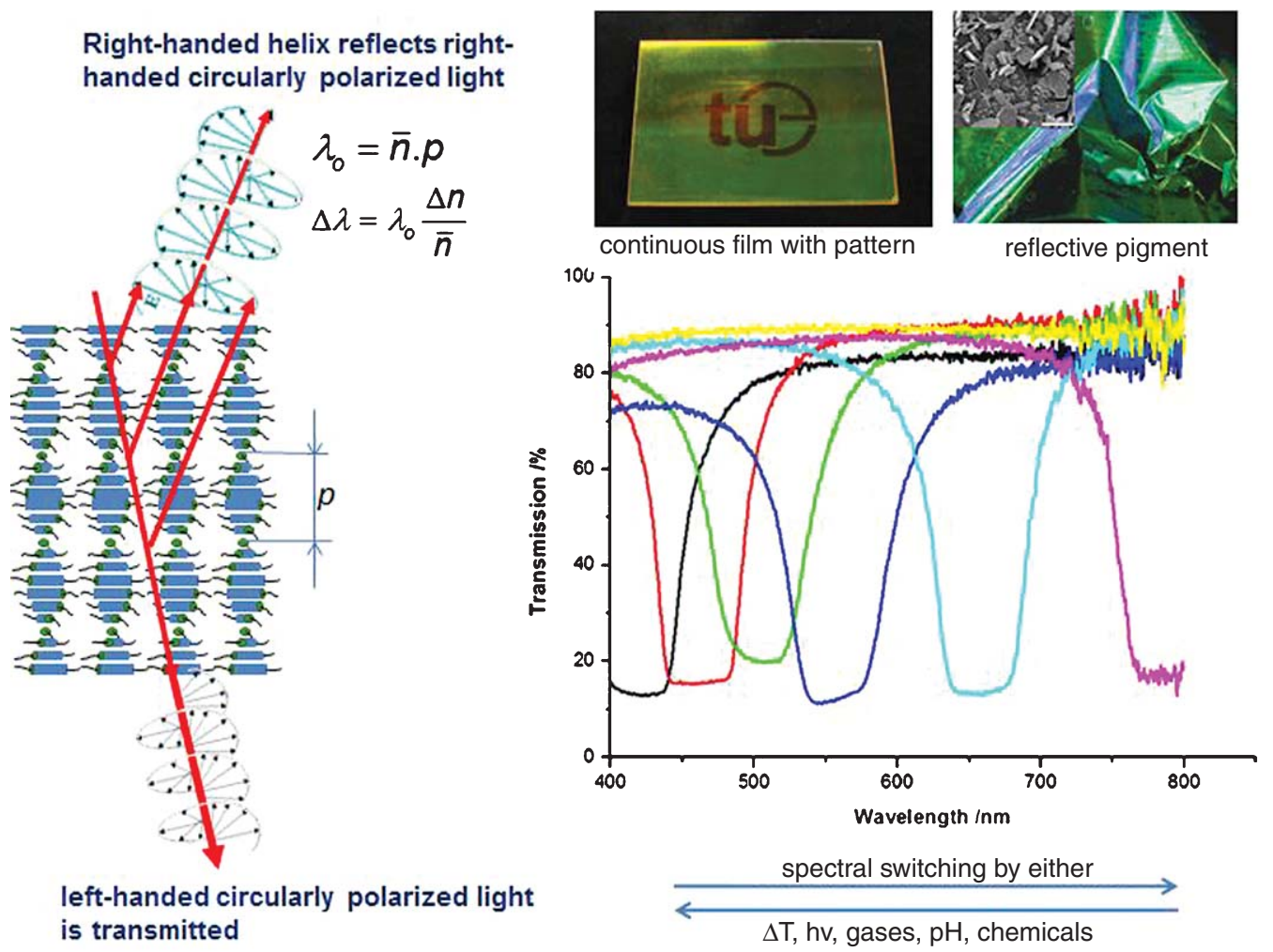

Fig. 2. Liquid crystal polymer networks with a helicoidal molecular order reflect light following Bragg's reflection rules. The reflection wavelength can be switched with a variety of triggers. The polymers can be applied as a continuous layer or as pigment added to a polymer binder.

small elements costly. To overcome this time-dimension paradox, self-organization of molecules helps to make these accurately-controlled structures available for large area applications in architecture. The display industry already smoothed this path in their production of optical films, which are used to improve television performance, basically defect-free by reel-to-reel processes.

\section{Morphing plastics}

The degree of molecular alignment of a liquid crystal network, usually defined by the order parameter quantifying the distribution of the rod-like molecular units along a common orientation (director), is currently 0.6 to 0.7 . Upon decreasing this order parameter, a uniaxially ordered polymer film tends to decrease its length in the direction along the molecular director and to expand in the two perpendicular directions. This property manifests itself during temperature cycling in a negative coefficient of thermal expansion along the director. By modulating the order parameter via external triggers, such as light or exposure to specific liquids or gases, liquid crystal networks can change their geometrical dimension and shape. These dimensional changes can be further tuned by employing non-unidirectional director profiles such as a molecular orientation following a twisted or a splay/bend configuration. These orientations force the plastic to bend in a controlled way. By utilizing even more 


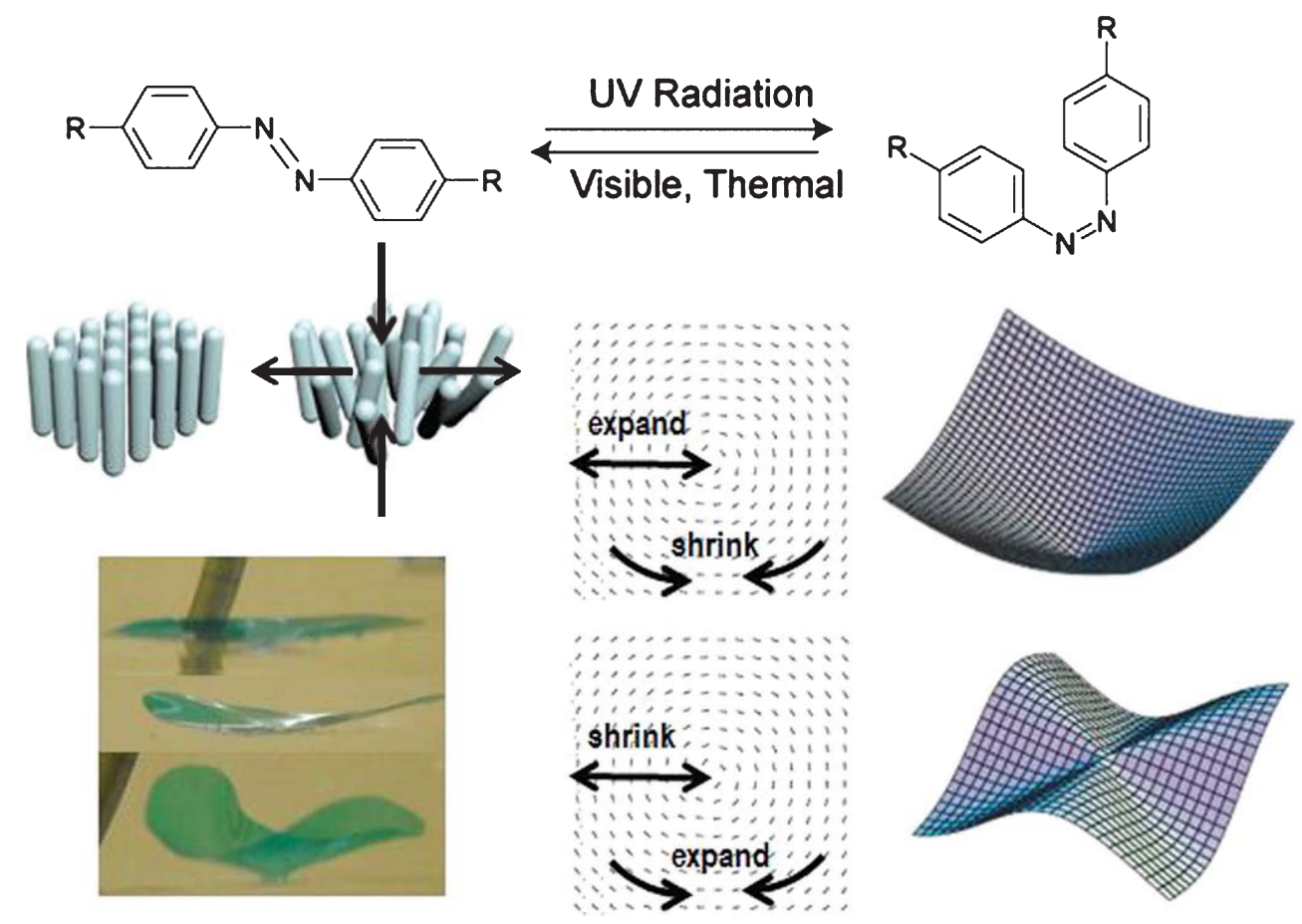

Fig. 3. An azo compound undergoes geometrical changes upon exposure to UV light. When integrated in a liquid crystal polymer network, this reaction changes the degree of order which, in turn, induces reversible geometrical changes. When the molecules are brought in an azimuthal orientation this process induces shape changes in a flat film leading to conical or anti-conical geometries which are experimentally verified. The depicted film was made by Carlos Sanchez (university of Zaragoza), and the deformation was modeled by Mark Warner9.

complex director designs, deformations can be made even more complex (Modes, 2011). For example, flat constructs with an azimuthal directors around a defect structure deform into a cone or into an anti-cone depending on whether the order parameter increases or decreases (Fig. 3).

An elegant way to change the order parameter is to introduce photo-sensitive groups into the liquid crystal network. Most well-known are azo moieties that reversibly change their conformation upon absorption of light (Fig. 3). Typically the azo compounds absorb light in the UV part of the spectrum. When exposed to $360 \mathrm{~nm}$ light they cause a liquid crystal network to deform in a manner dictated by its director pattern. When exposed with visible light they deform back into their initial shape, a process which also occurs spontaneously, albeit more slowly, in the dark via thermal processes. The chemistry behind liquid crystal network formation leaves much freedom for incorporating special properties. Rather than having the plastic morph in response to light or heat, one can also make them sensitive for water or water vapor, special chemicals, changes in $\mathrm{pH}$, or other stimuli (Harris, 2005, 2006). Control over the local molecular alignment will make it possible to switch between complex, pre-determined geometries.

As an alternative to shaping the geometry of a film or an object, one can use similar processes to morph the surface topology of a film or coating (Sousa, 2006). This is based on similar processes, changing the order parameter of the liquid crystal networks in patterned structures. Figure 4 demonstrates a coating consisting of patterned chiral-nematic in an isotropic liquid crystal sea. The surface 

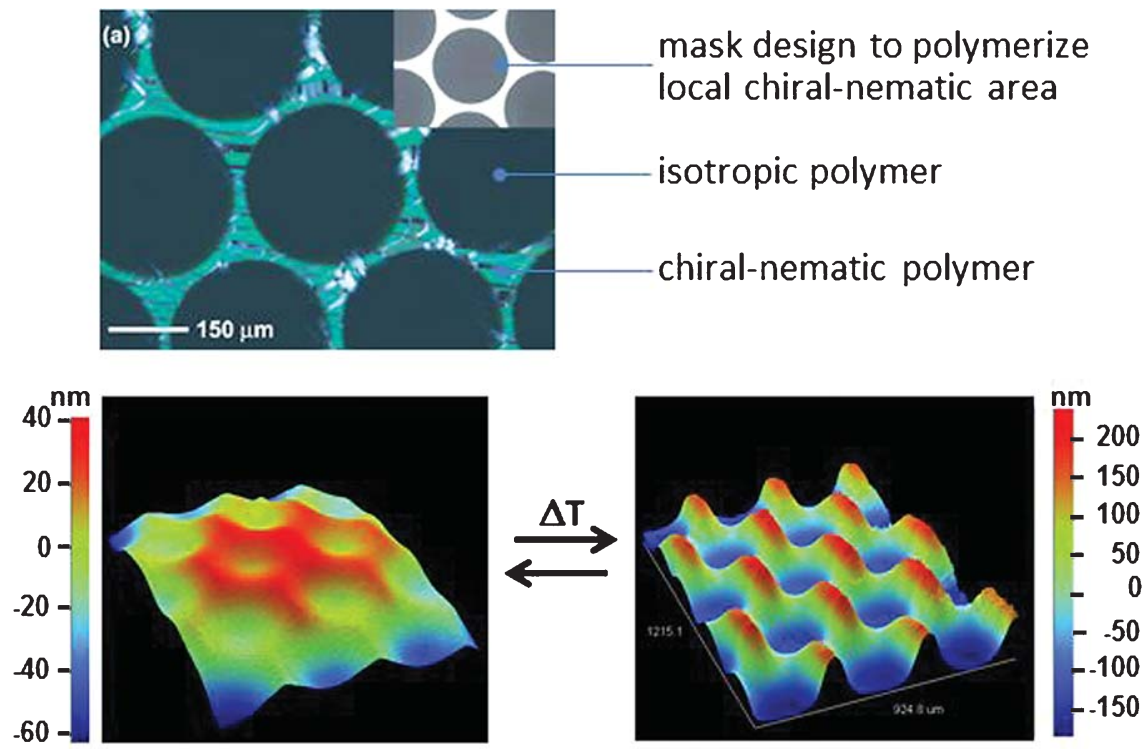

Fig. 4. A patterned surface coating containing both chiral nematic and isotropic regions can be made by local polymerization at two different temperatures. Decreasing the order parameter in the chiral-nematic area by raising the temperature makes them expand more than the isotropic area thus amplifying a surface relief structure.

topology alters through reversibly changing the order parameter. In this case, the surface structures were formed by temperature changes, but similar effects have been demonstrated using light-induced actuation. As an example of an application, the surface relief can be utilized to control the distance between two plate elements, thereby controlling heat transfer and thermal insulation.

\section{Nanoporous plastics}

The liquid crystal networks discussed so far are based on nematic liquid crystal order. In the nematic phase, on average the molecules are oriented in the same direction (which might rotate in case of the chiral systems) but they don't have a strict positional order. It is also possible to polymerize the liquid crystal monomers from a smectic phase. In the smectic phase, the monomeric entities adapt, positional ordering such that they are organized in layers in addition to their directional order. The thickness of these layers is equal to the length of the molecules, or even smaller in the case where the molecules interdigitate. Typically, this forms polymer films with periodic structures with lengths on the order of 3 nanometers. These smectic polymer networks are used to make thin, thermally stable polarizer films, for example.

Normally, monomers that form liquid crystal networks consist of atoms that are completely connected by strong covalent bonds. But chemistry also allows us to build in these monomers a weaker, so-called secondary bond. This weaker bond can be easily broken selectively, such as by temperature or through contact with and alkaline or acidic solution. An example of secondary bonds are those based on hydrogen bridges which are stable at moderate temperatures and $\mathrm{pH}$ conditions but open by treatment with a $\mathrm{pH}>9$ buffer or by heating to $160^{\circ} \mathrm{C}$. Through this modification the smectic 

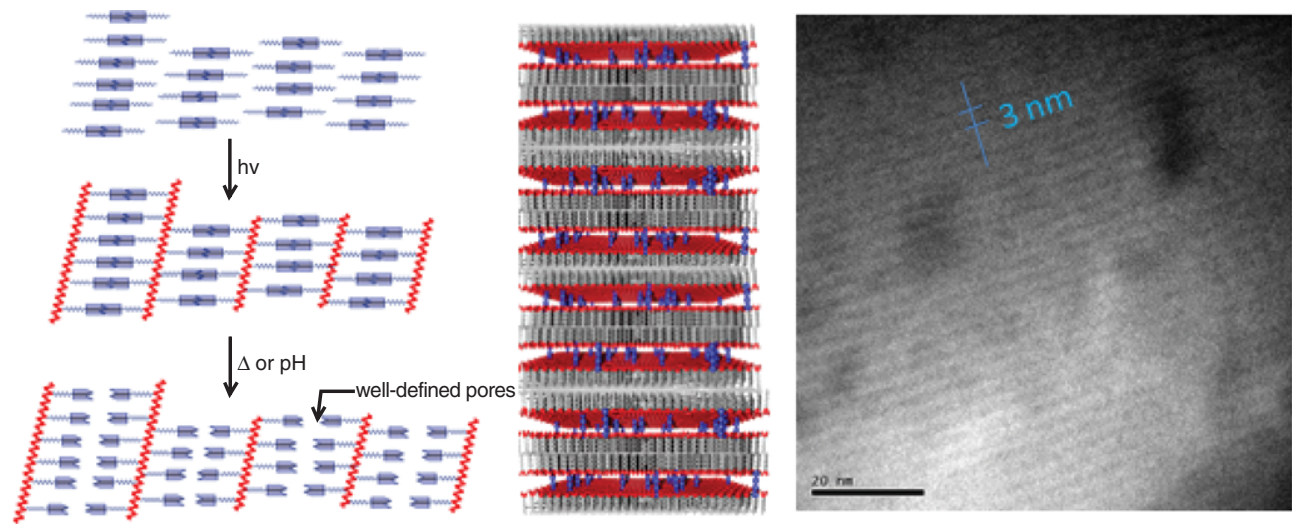

Fig. 5. Schematic representation of the formation of nanoporous plastic by polymerization of a smectic liquid crystal containing weak bonds. A two-dimensional porous structure is formed after breaking the weak bonds, e.g. by dipping in a moderately alkaline solution. The layers are kept together by fully covalent molecules. The cryo-TEM picture shows the nanopores after they are filled with barium ions for contrast.

layers in the polymer network can break-up into separated layers (Fig. 4). The integrity of the film is maintained by the presence of a small concentration of fully covalent smectic monomeric entities. These entities determine to what extent the smectic layers may separate, and their length determines the dimensions of the pores that are formed, which are typically in the range of 1 nanometer. The nanoporous polymers are being designed for size-exclusion filters and, can be made to 'breathe' air or liquid by peristaltic motion.

\section{Conclusions and outlook}

The combination of top-down structuring techniques like printing, lithography or embossing combined with bottom-up technologies such as self-organization of organic molecules has proven its value for applications in the world of electro-optics. In particular, the optics of flat panel television display has benefitted from liquid crystal technologies where molecular organization is controlled over large surface areas. It has now become worthwhile to study whether the extraordinary properties of liquid crystal networks can be used in the architectural world. The well-controlled molecular organization leads to autonomously changing properties in response to changes in the environment. Chiral networks can reversibly switch from transparent to reflective states, either in the visible or in the infrared part of the spectrum, enabling in-house climate control without compromising properties of window transparency or façade appearance. Autonomous morphing of films or coating surfaces can be used to control light capture by buildings and solar cells or to regulate heat transfer and thermal insulation. Nanoporous polymers may contribute to regulation of oxygen and $\mathrm{CO}_{2}$ levels in buildings while chiral networks may for instance indicate the presence of excessive $\mathrm{CO}_{2}$ concentrations.

However, these materials are new in architecture and civil engineering applications and need to prove their worth with respect to durability under harsh outdoor conditions. New concepts need to be developed, for integration in familiar architectural concepts such as paints, glass coatings and panels. But these new concepts can also be considered for more active elements for indoor textiles that react to climate changes or sun position, thus connecting outdoor conditions with in-house appearance. 


\section{References}

Broer, D. J., Lub, J., \& Mol, G. N. (1995). Wide-band reflective polarizers from cholesteric polymer networks with a pitch gradient. Nature, 378(6556), 467-469.

Broer, D. J., Crawford, G. P., \& Zumer, S. (2011). Crosslinked Liquid Crystalline Networks - From Rigid Polymer Networks to Elastomers, CRC Press Taylor \& Francis group, New York.

Yamada, M., Kondo, M., Mamiya, J.-I., Yu, Y., Kinoshita, M., Barrett, C. J., \& Ikeda, T. (2008). Photomobile polymer materials - towards light-driven plastic motors. Angew Chem Int Ed, 47, 4986-4988. Macromolecules, 41(22), 8592-8596.

Han, Y., Pacheco, K., Bastiaansen, C. W. M., Broer, D. J., \& Sijbesma, R. P. (2010). Optical monitoring of gases with cholesteric liquid crystals. J Am Chem Soc 132, 2961-2967.

Harris, K. D., Bastiaansen, C. W. M., Lub, J., \& Broer, D. J. (2005). Self-assembled polymer films for controlled agent-driven motion. Nano Letters 5(9), 1857-1860.

Harris, K. D., Bastiaansen, C. W. M., \& Broer, D. J. (2006). A glassy bending-mode polymeric actuator which deforms in response to solvent polarity. Macromol Rapid Commun, 27, 1323-1329.

Klein, T. (2011) Outcome of brainstorm meetings with participants of Delft University of Technology and Eindhoven University of Technology. Luengo Gonzalez, C., Bastiaansen, C. W. M., Lub, J., Loos, J., Lu, K., Wondergem, H. J., \& Broer, D. J. (2008). Nanoporous membranes made of hydrogen bridged smectic networks with nanometer transverse pore dimensions. Advanced Materials 20(7), 1246-1252.

Modes, C. D., Bhattacharya, K., \& Warner, M. (2011). Gaussian curvature from flat elastic sheets. Proc R Soc A 467, 1121-1140.

Sousa, M. E., Broer, D. J., Bastiaansen, C. W. M., Freund, L. B., Crawford, G. P. (2006). Isotropic Islands in a cholesteric sea: Patterned thermal expansion for responsive surface topologies. Adv Mat 18(14), 1842-1845.

Van Oosten, C. L., Bastiaansen, C. W. M., \& Broer, D. J. (2009). Printed artificial cilia from liquid-crystal network actuators modularly driven by light. Nature Materials 8(8), 677-682.

Van Oosten, C. L., Corbett, D., Davies, D., Warner, M., Bastiaansen, C. W. M., \& Broer, D. J. (2008). Bending Dynamics and Directionality Reversal in Liquid Crystal Network Photo-actuators. 